\title{
The effect of exercise training and physiotherapy on diastolic function, exercise capacity and quality of life in patients with heart failure with preserved ejection fraction: a systematic review and meta-analysis
}

\author{
Chenchen Zhuang ${ }^{1 *}$, Xufei Luo*, Qiongying Wang' ', Wenjuan Wang' ${ }^{1}$, Runmin Sun'1, Xiaofang Zhang' ${ }^{1}$ Jing Yu \\ 'Hypertension Center, Lanzhou University Second Hospital, Lanzhou, China \\ ${ }^{2} S$ chool of Public Health, Lanzhou University, Lanzhou, China \\ *These authors contributed equally to this work
}

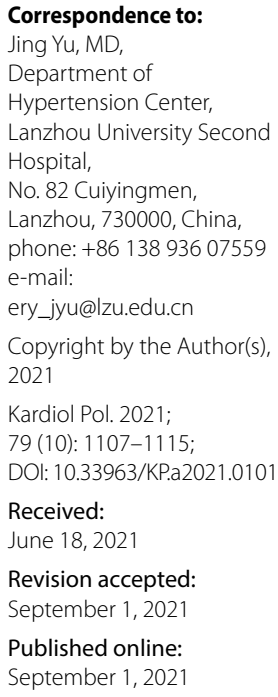

\begin{abstract}
A B S T R A C T
Background: Exercise and physiotherapy are accepted as an important contribution to the rehabilitation of patients with heart failure with preserved ejection fraction (HFpEF). But the previous results are unclear partly because of their limited power and small sample sizes.
\end{abstract}

Aims: We aimed to understand better the effects of two exercise training interventions and two modalities of physiotherapies on exercise capacity, quality of life (QoL), and diastolic dysfunction in HFpEF patients.

Methods: The Cochrane Library, EMBASE, and MEDLINE via PubMed were searched for randomized controlled trials from their inception to May 2021. The effect size was estimated as mean differences (MD) with 95\% confidence intervals (Cl).

Results: A total of 14 articles on 13 trials were included in this meta-analysis with $673 \mathrm{HFpEF}$ patients. The pooling revealed that peak oxygen uptake was improved by endurance training, functional electrical stimulation (FES), and inspiratory muscle training (IMT). Similar results were observed for a 6-minute walk test and QoL. A combination of endurance and resistance training (combined exercise) was beneficial to the ratio of peak early to late diastolic mitral inflow velocities (MD [95\% CI]:-2.90 $[-4.97,-0.83] ; P=0.006$ ) and the early diastolic mitral annual velocity (MD [95\% Cl]: $1.40[0.68,2.12] ; P=0.006]$. IMT improved the ventilation/carbon dioxide ratio slope (MD [95\% Cl]: $-3.36 \mathrm{ml} / \mathrm{kg} / \mathrm{min}[-6.17,-0.54] ; P=0.019]$.

Conclusions: FES and IMT improve functional capacity and QoL without a change in diastolic function in HFpEF patients, and the outcomes are similar to endurance training. Notably, combined exercise may improve diastolic function.

Key words: diastolic function, exercise training, functional electrical stimulation, heart failure with preserved ejection fraction, inspiratory muscle training

Kardiol Pol 2021; 79, 10: 1107-1115

\section{INTRODUCTION}

Heart failure with preserved ejection fraction (HFpEF) accounts for nearly half of heart failure (HF) patients in the community, and the mortality and morbidity are high [1]. However, the established neurohormonal-based therapies, used for treating heart failure with reduced ejection fraction (HFrEF), have failed to improve exercise intolerance and provide favorable clinical outcomes for HFpEF [2]. The study of Lelonek [1] reported that although angiotensin receptor neprilysin inhibitors (ARNIs) may benefit from treatment with HFpEF, the relevance of ARNI in HFpEF still has not been clarified.

Cardiac dysfunction appears in diastolic dysfunction in echocardiography, exercise intolerance may be objectively measured by peak oxygen uptake (peak $\mathrm{VO}_{2}$ ), and poor quality of life (QoL) completes the typical clinical image of HFpEF [3]. Exercise training appears to be a promising strategy to improve peak $\mathrm{VO}_{2}$ and QoL in HFpEF patients [4, 5]. However, previous meta-analyses hold inconsistent opinions on diastolic function 
WHAT'S NEW?

Exercise and physiotherapy are accepted as an important contribution to the rehabilitation of patients with heart failure with preserved ejection fraction (HFpEF). This study aimed to evaluate the effect of endurance training and a combination of endurance and resistance training (combined exercise), functional electrical stimulation (FES), and inspiratory muscle training (IMT) on HFpEF patients, measured by diastolic function, exercise capacity, and quality of life. This is the first study to evaluate different modalities of physical therapy on HFpEF patients. Our results showed that FES and IMT, as well as endurance training, improved functional capacity and quality of life (QoL). Moreover, combined exercise was beneficial to the ratio of peak early to late diastolic mitral inflow velocities and the early diastolic mitral annual velocity. These findings suggest that FES and IMT improve functional capacity and QoL in HFpEF patients, and the outcomes are similar to endurance training. Additionally, combined exercise may improve diastolic function.

in HFpEF patients experiencing exercise training [6-8] partly due to their failure to assess different modalities of exercise training.

$\mathrm{HFpEF}$ is more common in elderly patients, and these patients have poor adherence to exercise training [9]. It is time to carry out physiotherapy to relieve the symptoms of HFpEF. At present, physiotherapy mainly includes inspiratory muscle training (IMT) and functional electrical stimulation (FES), which are effective interventions to improve exercise intolerance in $\operatorname{HFrEF}[10,11]$. HF has been commonly associated with inspiratory muscle weakness [12]. IMT, a training stimulus directly to the inspiratory muscles, improves inspiratory muscle weakness, cardiorespiratory fitness, and QoL, like exercise training, leading a better adaptation to posterior exercise training [13]. The other physiotherapy involves a neuromuscular stimulation FES, which delivers a specific recruitment pattern for performing a muscular movement necessary for exercise [14]. FES has shown potential beneficial effects in HF patients, including increased muscle mass and improved QoL $[15,16]$. IMT and FES may serve HF patients excluded from exercise training and constitute interesting treatment options for clinicians. Accordingly, both IMT and FES may have potential benefits to HFpEF patients, which may not only be limited in HFpEF patients unable to undergo exercise training but also may be extended to the general HFpEF population. However, so far there have been no published meta-analyses to evaluate the impact of IMT and FES on exercise tolerance in HFpEF patients. In this meta-analysis, we aimed to evaluate the effects of different modalities of exercise training (endurance, and a combination of endurance and resistance training, combined exercise), and physiotherapies (FES and IMT) on exercise capacity, QoL, and diastolic function in HFpEF patients.

\section{METHODS}

The present study was conducted in accordance with the Preferred Reporting Items for Systematic Reviews and Meta-Analyses [17]. The protocol was prospectively registered with Open Science Framework (https://osf.io/sufc8).

\section{Search strategy}

The studies on the effect of physical therapy in HFpEF patients published before May 20, 2021 were searched in the Cochrane Library, EMBASE, and MEDLINE via PubMed. We used a mix of medical subject headings (MeSH) and keywords including exercise training, aerobic exercise, endurance training, inspiratory muscle training, functional electrical stimulation, and heart failure with preserved ejection fraction.

\section{Study selection}

Studies were considered eligible if they (1) were published as randomized controlled trials (RCTs); (2) included patients (aged $\geq 18$ years) with HFpEF; (3) included patients undergoing physical therapy; (4) included a comparison of physical therapy with standard medical care or placebo control group. Articles that failed to meet the inclusion criteria were removed, including reviews, animal studies, non-RCTs, non-English language, and intervention duration of fewer than 4 weeks.

\section{Main outcomes}

The primary outcomes of this study were exercise capacity measured by peak $\mathrm{VO}_{2}$ and a 6-minute walk test (6MWT), QoL measured by the Minnesota Living with Heart Failure Questionnaire (MLHFQ) total score. Secondary outcomes were evaluated by the ventilation/carbon dioxide ratio slope $\left(\mathrm{VE} / \mathrm{VCO}_{2}\right)$ and diastolic function, measured by peak early to late diastolic mitral inflow velocities $(E / A)$, the ratio of early diastolic mitral inflow to annular velocities $\left(E / e^{\prime}\right)$, and the early diastolic mitral annular velocity $\left(e^{\prime}\right)$.

\section{Data extraction}

Two reviewers (CCZ and XFL) extracted the following data independently: study characteristics (authors, year of publication, and country), participant characteristics (age and sample size of different groups), study methods/design, and the period of exercise intervention.

\section{Risk of Bias}

We evaluated the risk of bias for inclusion in this metaanalysis by the Physiotherapy Evidence Database (PEDro) 
scale [18]. When a disagreement occurred, a third reviewer was consulted.

\section{Statistical analysis}

For each outcome, the effect size in our study was assessed by change from the baseline to follow-up. We used weighted mean difference (MD) and 95\% confidence intervals (Cl) for the same scale of the outcomes. When the ${ }^{2}$ statistic was lower than $30 \%$ and $P<0.10$, a fixed-effect model was used; otherwise, a random-effect model was used. All analyses were used by STATA version 14.0 (StataCorp, College Station, TX, USA). Furthermore, meta-regression analyses were also performed with STATA software using the restricted maximum likelihood method. Two dependent variables (peak $\mathrm{VO}_{2}$ and QoL) were tested against several independent variables including age, sex, and an exercise period. A value of $P<0.01$ was considered significant.

\section{RESULTS}

\section{Included studies}

The flow chart is shown in Figure 1, screening identified 129 potential reports. After the removal of duplicates, 152 records remained. 117 studies were excluded after scanning titles and abstracts. Then 21 were excluded because they did not report HFpEF. Ultimately, 11 RCTs on exercise training $[5,19-28]$ and 3 RCTs on physiotherapies were included in this meta-analysis [14, 29, 30].

\section{Characteristics of studies}

The basic characteristics of each study were summarized in Table S1. The present meta-analysis included $673 \mathrm{HF}$ -
-pEF patients. The mean age of participants ranged from 60.5 to 75 years, and the proportion of men ranged from $0 \%$ to $64 \%$.

\section{Quality assessment}

The quality of included RCTs is presented in Supplementary material, Table S2. In none of the studies was there objective evidence of imbalance in the baseline characteristics between the intervention and control groups. The moderate risk of bias was due to inadequate blinding of participants and therapists, allocation concealment, and intention-to-treat methodologies.

\section{Functional capacity indicator}

Our meta-analysis could be performed for two functional capacity indicators: a $6 \mathrm{MWT}$ and peak $\mathrm{VO}_{2}$. Eight trials with 411 patients reported on 6MWT (Figure $2 \mathrm{~A}$ ). The heterogeneity was small $\left(I^{2}=0.0 \% ; P=0.560\right.$, fixed-effect). The 6MWT was increased by endurance training (MD $[95 \% \mathrm{Cl}]$ : $38.79 \mathrm{~m}$ [19.97, 57.61]; $P<0.001)$ and FES (MD [95\% Cl]: $52.77 \mathrm{~m}[30.61,74.93]$; $P<0.001)$. However, there was no change after IMT (MD [95\% CI]: $84.00 \mathrm{~m}$ [-31.73, 199.73]; $P=0.155)$ and combined exercise (MD [95\% Cl]: $7.00 \mathrm{~m}$ $[-37.61,51.61] ; P=0.758)$.

Thirteen trials with 411 patients reported on peak $\mathrm{VO}_{2}$ (Figure $\left.2 \mathrm{~B}\right)$. The heterogeneity was small $\left(R^{2}=0.0 \%\right.$; $P=0.739$, fixed-effect). Peak $\mathrm{VO}_{2}$ was improved by endurance training (MD [95\% Cl]: $1.89 \mathrm{ml} / \mathrm{kg} / \mathrm{min}[1.32,2.46]$, $P<0.001)$, FES (MD [95\% Cl]: $2.28 \mathrm{ml} / \mathrm{kg} / \mathrm{min}[0.92,3.65]$; $P=0.001$ ), IMT (MD [95\% Cl]: $2.72 \mathrm{ml} / \mathrm{kg} / \mathrm{min}[1.44$, 3.99]; $P<0.001)$ and combined exercise (MD [95\% Cl]: $3.30 \mathrm{ml} / \mathrm{kg} / \mathrm{min}[0.44,6.16] ; P=0.024)$. Meta-regression

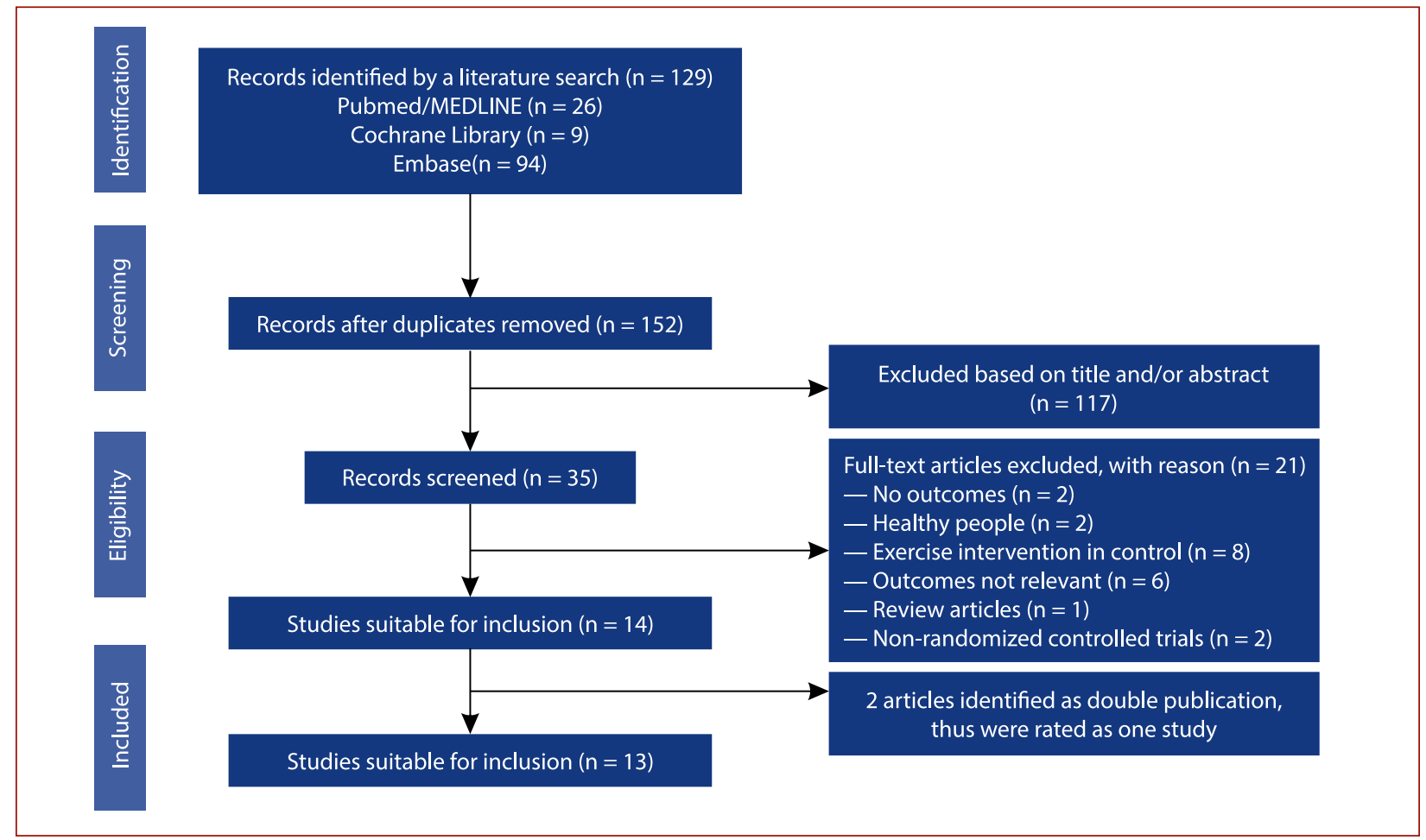

Figure 1. Flow chart of the study selection procedure 


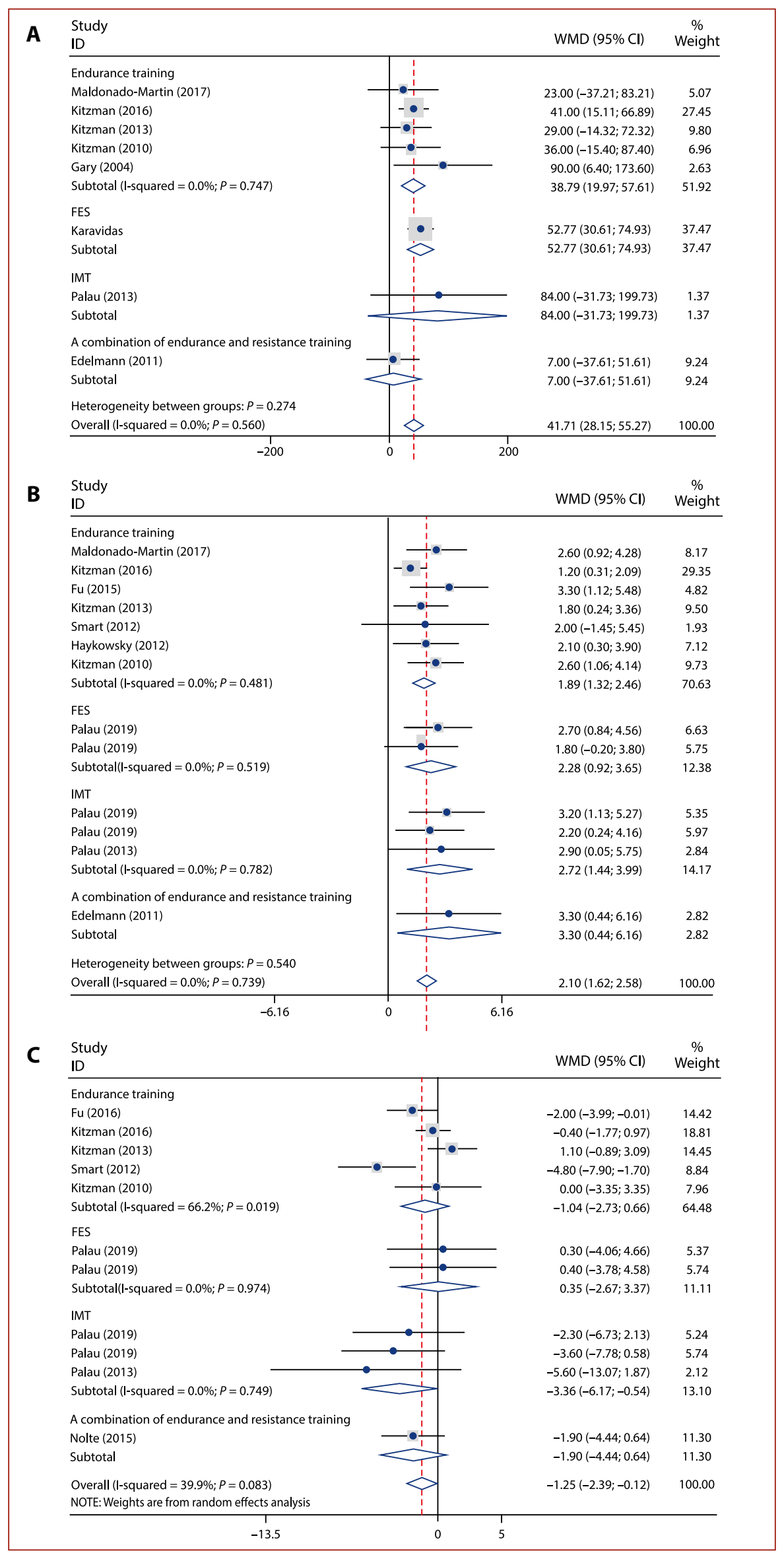

Figure 2. A. Effects of exercise on exercise performance: 6-minute walk test (6MWT). B. Change in peak oxygen consumption (peak VO${ }_{2}$ ), ventilation/carbon dioxide ratio slope $\left(\mathrm{VE} / \mathrm{VCO}_{2}\right.$ slope $)$ 


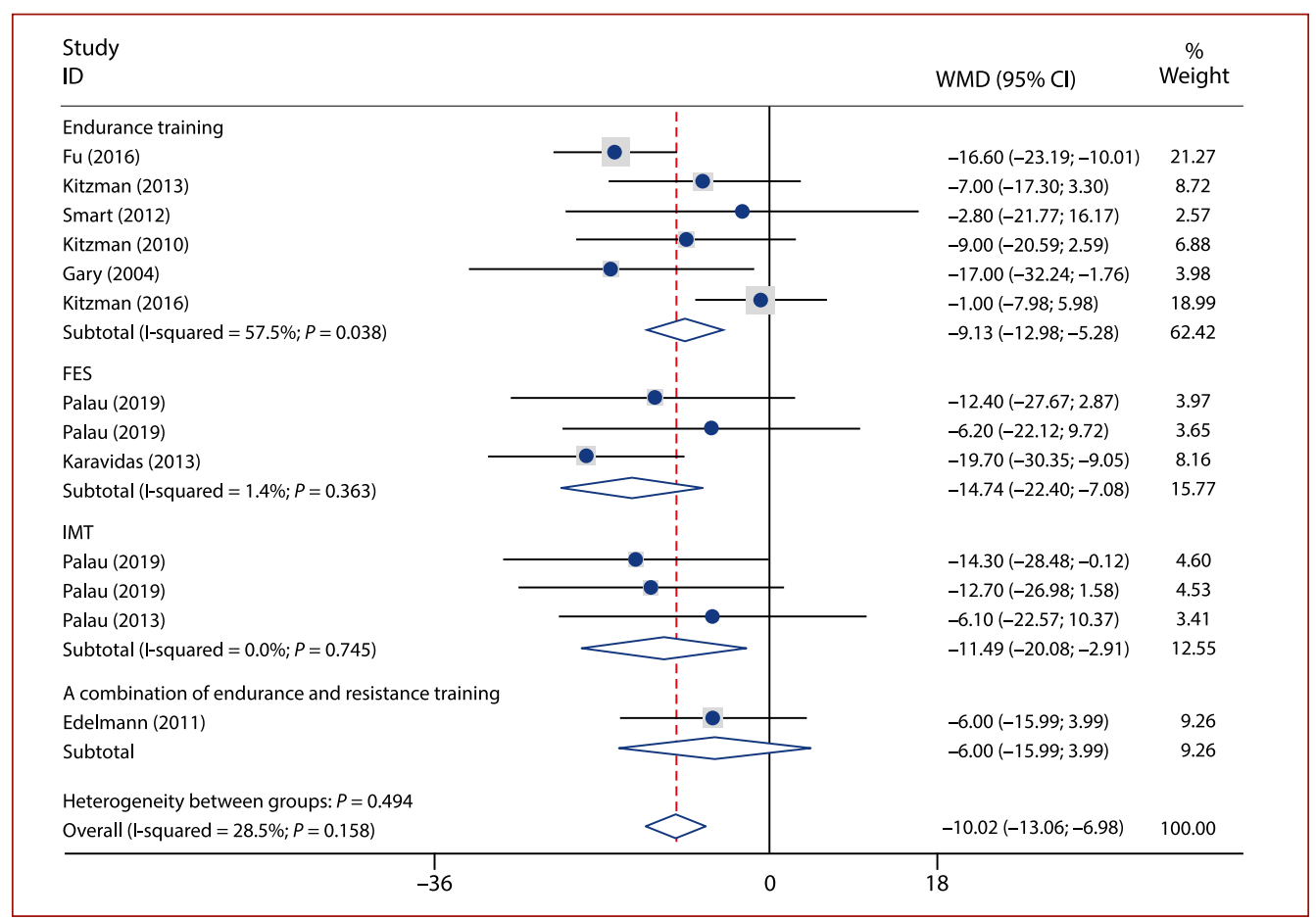

Figure 3. Effects of exercise on Minnesota Living With Heart Failure Questionnaire (MLHFQ) total score

analysis showed no significant effect of age (coefficient, $0.007 ; P=0.989$ ), sex (coefficient, $0.010 ; P=0.826$ ), or exercise period (coefficient, $-0.736 ; P=0.036$ ).

\section{Quality of life}

Thirteen trials with 560 patients reported on the MLHFQ total score (Figure 3$)$. The heterogeneity was small $\left(I^{2}=28.5 \%\right.$; $P=0.158$, fixed-effect). The MLHFQ total score was improved by endurance training (MD [95\% Cl]: -9.13 [-12.98, -5.28]; $P<0.001]$, FES (MD [95\% Cl]: - 14.74 [-22.44, -7.08]; $P<0.001)$ and IMT (MD [95\% Cl]: -11.49 [-20.08, -2.91]; $P=0.009)$. However, no difference was found in the case of combined exercise (MD [95\% Cl]: -6.00 [-15.99, 3.99]; $P=0.239$ ). Meta-regression analysis showed no significant effect of exercise period (coefficient, 1.116; $P=0.574$ ), age (coefficient, $-0.689 ; P=0.800$ ), or sex (coefficient, -0.523 ; $P=0.852)$.

\section{Diastolic function}

Eight trials with 416 patients reported on E/A (Figure 4A). The heterogeneity was small $\left(R^{2}=0 \% ; P=0.606\right.$, fixed-effect). E/A was no change by endurance training (MD [95\% $\mathrm{Cl}]: 0.03[-0.03,0.09] ; P=0.307)$, combined exercise (MD $[95 \% \mathrm{Cl}]:-0.03[-0.17,0.11] ; P=0.678)$, and FES (MD [95\% Cl]: $-0.12[-0.29,0.05] ; P=0.162)$.

Ten trials with 416 patients reported on E/e' (Figure 4B). There was a statistical heterogeneity $\left({ }^{2}=43.1 \% ; P=0.071\right.$, random-effect). E/e' was improved by combined exercise with one included study (MD [95\% Cl]: -2.90 [-4.97, -0.83]; $P=0.006)$. There was no change in $E / e^{\prime}$ by endurance training (MD [95\% Cl]: $-0.03[-2.83,2.78] ; P=0.983)$, FES (MD
[95\% Cl]: $-2.16[-4.41,0.09] ; P=0.060)$ and IMT (MD [95\% $\mathrm{Cl}]:-1.10[-4.56,2.36] ; P=0.533)$.

Four trials with 215 patients reported on e' (Figure 4C). There was a statistical heterogeneity $\left(I^{2}=81.3 \% ; P=0.001\right.$, random-effect). $e^{\prime}$ was improved by combined exercise in one included study (MD [95\% Cl]: $1.40[0.68,2.12] ; P<0.001$ ). There was no change in e' by endurance training (MD $[95 \%$ $\mathrm{Cl}]:-2.90[-4.97,-0.83] ; P=0.140)$ and IMT (MD [95\% CI]: $0.30[-1.28,1.88] ; P=0.709)$.

\section{Exercise physiology parameter}

Eleven trials with 502 patients reported on the VE/ $\mathrm{VCO}_{2}$ slope (Figure 2C). There was a statistical heterogeneity $\left(I^{2}=39.9 \%\right.$; $P=0.083$, random-effect). The VE/VCO 2 slope was improved by IMT (MD [95\% CI]: $-3.36[-6.17,-0.54] ; P=0.019$ ). No significant difference was found after endurance training (MD [95\% Cl]: - $1.04[-2.73,0.64] ; P=0.226)$, FES (MD [95\% $\mathrm{Cl}]: 0.35[-2.67,3.37] ; P=0.819)$ or combined exercise (MD [95\% Cl]: $-1.90[-4.44,0.64] ; P=0.142)$. Smart et al. [22] may be the source of heterogeneity due to the small sample.

\section{DISCUSSION}

The present meta-analysis summarized data that evaluated the effects of two exercise interventions and two physiotherapy modalities in HFpEF patients. FES and IMT improved exercise performance and QoL, and the outcomes were similar to endurance training. Notably, combined exercise has the potential to improve diastolic function in HFpEF patients.

The previous studies have focused on overall exercise training rather than the different types of exercise training. 


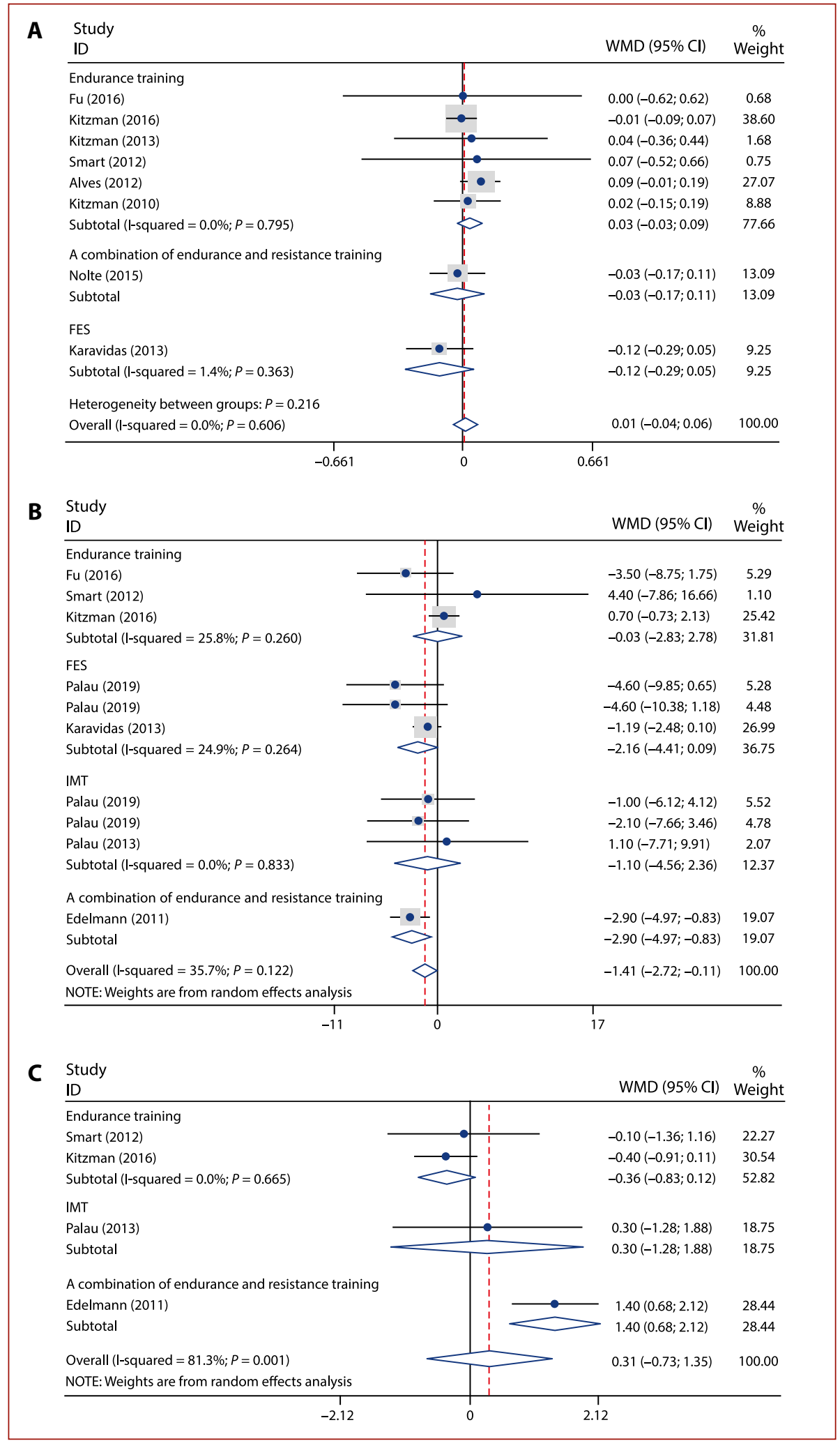

Figure 4. Effects of exercise on diastolic function: A. the ratio of peak early to late diastolic mitral inflow velocities (E/A). B. Change in the ratio of early diastolic mitral inflow to annular velocities $\left(E / E^{\prime}\right)$. C. The early diastolic mitral annular velocity $\left(e^{\prime}\right)$

The meta-analysis of Gomes-Neto et al. [31] only conducted endurance training and failed to represent other exercise modalities. Some meta-analyses $[8,32]$ showed that exercise training improved exercise capacity and QoL in HFpEF patients, but a methodological limitation was the combination of endurance training and combined exercise. The previous meta-analyses held inconsistent opinions on diastolic function in HFpEF patients during exercise training $[6-8,32]$. Thus, the present meta-analysis compensated for the weakness of the previous studies. 
The present meta-analysis observed that peak $\mathrm{VO}_{2^{\prime}}$ $6 \mathrm{MWT}$, and QoL were improved by exercise training, as well as FES and IMT. The peak $\mathrm{VO}_{2}$ may affect oxygen delivery and/or utilization via cardiac, vascular, and skeletal muscle function [33]. Therefore, active skeletal muscle is the major reason to induce the augment of peak $\mathrm{VO}_{2}$ in HFpEF patients, including oxidative enzyme activity and capillary density [25].

Left ventricular (LV) diastolic dysfunction plays a pivotal role in the pathophysiological hallmark of HFpEF [34]. In assessing patients for diastolic dysfunction and possible $\mathrm{HFpEF}$, the use of the 2009 consensus guideline on diastolic function yielded a sensitivity of $47 \%$ to rule out $\mathrm{HFpEF}$, whereas other proposed classification schemes actually had higher sensitivities between $72 \%$ and $77 \%$ to rule out $\operatorname{HFpEF}[35,36]$. Notably, echocardiography for impaired diastolic dysfunction has been the sine qua non of diagnosis in HFpEF [37]. Earlier occurring LV diastolic pressure relates best with $E / A, E / e^{\prime}$, and $e^{\prime}[38]$. With respect to the estimation of LV filling pressures, early work suggested that $E / e^{\prime}$ could be used to estimate reliably the LV filling pressure in HFpEF and even in atrial fibrillation. The E-wave is smaller, leading to the diastolic dysfunction filling pattern, where $E / A<1$, occurring with hypertension, hypertrophic cardiomyopathy, ischemia, and myocardial infarction. Reduced e'velocity results from a variety of comorbidities related to impaired myocardial relaxation and restoration forces. Edelmann et al. [19] reported that combined exercise improved diastolic function. Our findings showed that combined exercise improved $E / e^{\prime}$ and e', suggesting an improvement in left ventricular filling pressures. However, endurance training failed to improve diastolic function. Smart et al. [22] suggested that 16-week endurance training may not be sufficient to elicit alteration in myocardial properties. Fujimoto et al. [39] reported that 1-year endurance training had little effect on left ventricular compliance in HFpEF patients. In response to exercise training, cardiac relaxation may be compounded by abnormalities in skeletal muscle oxygen use, which augments cardiac output and flow into a small, stiff, and slowly relaxing heart [40].

The hemodynamic changes that occur during exercise constitute the primary stimulus for diastolic function [41]. Endurance training sustained elevations in cardiac output with reduced peripheral vascular resistance by an increased mitochondrial biogenesis and capillary density, aiding in the transport and use of oxygen to generate energy [42]. Resistance intense bouts of increased peripheral vascular resistance and only slightly evaluated cardiac output by training add muscle bulk to peripheral muscles and increase bone mass, leading in turn to an increase in muscle strength and power [43]. Thus, the difference in diastolic function may be induced by resistance training.

FES and IMT had no substantial changes in the E/e', E/A, and e', despite the improvement in exercise capacity and QoL $[14,30]$. Extra-cardiac effects not related directly to an improvement in cardiac function may play a critical role in the beneficial effect of physiotherapy. Our result showed that IMT significantly improved the $\mathrm{VE} / \mathrm{VCO}_{2}$ slope alone. Various studies of HF patients have shown that selective respiratory muscle training improved submaximal and maximal exercise capacity during daily living activities [13].The development of diaphragmatic fatigue is delayed, leading to a reduction in the recruitment of accessory respiratory muscles, and an improved ventilatory efficiency [44]. Although FES fails to improve the VE/VCO slope, the beneficial effects of FES on functional capacity and QoL is similar to those of IMT. FES and IMT interventions improve the recruitment of accessory respiratory muscles and ventilatory efficiency by delaying diaphragmatic fatigue, which increases muscle strength, muscle mass, and aerobic-oxidative capacity $[10,29]$. Both IMT and FES interventions are simple, low-cost, and harmless for patients with HFpEF, and may serve as "bridge therapies" to exercise training.

Physical therapy may be a safe approach for the treatment of HFpEF. However, a demanding challenge for exercise training will be translating these programs to HFpEF patients with relevant comorbidity (i.e. frailty) after cardiac surgery or due to advanced age. Notably, FES and IMT seem to be alternative treatments for patients who are unable to perform exercise training. In fact, many patients with HF$\mathrm{pEF}$, unable to exercise, pay more attention to medication treatments. Thus, the greatest challenge is to implement the existing knowledge about training benefits in HFpEF as a standard in clinical practice and to increase participation rates of patients with a clear indication for physiotherapy-based cardiac rehabilitation in existing programs.

\section{Strengths and limitations}

This study is the first to evaluate different modalities of exercise training and physiotherapy on exercise capacity, QoL, and diastolic function in HFpEF patients. However, several limitations should be addressed. Firstly, the major limitation of this analysis is the small sample size in most of the RCTs. There were only two studies that reported combined exercise, and they shared one set of data using different results $[19,20]$. New large-scale RCTs are needed to confirm the findings of this meta-analysis. Secondly, few studies compared exercise training and physiotherapy to exercise capacity, QoL, and diastolic function. Accordingly, it is not possible to asess which intervention modality would be most beneficial for HFpEF patients. Thirdly, heterogeneity scores suggested the majority of analyses were justified, but this of e' may display heterogeneity at levels too high to justify this analysis. Finally, more standardized, high-quality? qualitative, larger-scale, and longer intervention trials are needed in order to estimate the most effective training modality.

\section{CONCLUSIONS}

Our meta-analysis suggests that FES and IMT, as well as endurance training, have a positive effect on functional 
capacity and QoL without causing a significant change in diastolic function. Notably, combined exercise may improve diastolic function and peak $\mathrm{VO}_{2}$. Further trials are required to determine which training modalities are effective forms of training to improve aerobic capacity in HFpEF patients.

\section{Article information}

Funding: This study was supported by the National Natural Science Foundation of China (NSFC 81960086), and the Cuiying Scientific and Technological Innovation Program of Lanzhou University Second Hospital (CY2017-BJ02).

Conflict of interest: None declared.

Open access: This article is available in open access under Creative Common Attribution-Non-Commercial-No Derivatives 4.0 International (CC BY-NC-ND 4.0) license, allowing to download articles and share them with others as long as they credit the authors and the publisher, but without permission to change them in any way or use them commercially. For commercial use, please contact the journal office at kardiologiapolska@ptkardio.pl.

How to cite: Zhang C, Luo X, Wang Q, et al. The effect of exercise training and physiotherapy on diastolic function, exercise capacity, and quality of life in patients with heart failure with preserved ejection fraction: a systematic review and meta-analysis. Kardiol Pol. 2021; 79(10): 1107-1115, doi: 10.33963/KP.a2021.0101.

\section{REFERENCES}

1. Lelonek M. Heart failure with preserved ejection fraction after the PARAGON-HF trial results: current knowledge and future directions. Kardiol Pol.2020; 78(12): 1199-1205, doi: 10.33963/KP.15639, indexed in Pubmed: 33021355.

2. Kaila K, Haykowsky MJ, Thompson RB, et al. Heart failure with preserved ejection fraction in the elderly: scope of the problem. Heart Fail Rev. 2012; 17(4-5): 555-562, doi: 10.1007/s10741-011-9273-z, indexed in Pubmed: 21818681.

3. Haykowsky M, Brubaker P, Kitzman D. Role of physical training in heart failure with preserved ejection fraction. Curr Heart Fail Rep. 2012; 9(2): 101-106, doi: 10.1007/s11897-012-0087-7, indexed in Pubmed: 22430146.

4. Dieberg G, Ismail H, Giallauria F, et al. Clinical outcomes and cardiovascular responses to exercise training in heart failure patients with preserved ejection fraction: a systematic review and meta-analysis. J Appl Physiol (1985). 2015; 119(6): 726-733, doi: 10.1152/japplphysiol.00904.2014, indexed in Pubmed: 25749444.

5. Kitzman DW, Brubaker P, Morgan T, et al. Effect of caloric restriction or aerobic exercise training on peak oxygen consumption and quality of life in obese older patients with heart failure with preserved ejection fraction: a randomized clinical trial. JAMA. 2016; 315(1): 36-46, doi: 10.1001/jama.2015.17346, indexed in Pubmed: 26746456.

6. Pandey A, Parashar A, Kumbhani D, et al. Exercise training in patients with heart failure and preserved ejection fraction: meta-analysis of randomized control trials. Circ Heart Fail. 2015; 8(1): 33-40, doi: 10.1161/CIRCHEARTFAILURE.114.001615, indexed in Pubmed: 25399909.

7. Chan $E$, Giallauria $F$, Vigorito $C$, et al. Exercise training in heart failure patients with preserved ejection fraction: a systematic review and meta-analysis. Monaldi Arch Chest Dis. 2016; 86(1-2): 759, doi: 10.4081/monaldi.2016.759, indexed in Pubmed: 27748473.

8. Fukuta $\mathrm{H}$, Goto $\mathrm{T}$, Wakami $\mathrm{K}$, et al. Effects of exercise training on cardiac function, exercise capacity, and quality of life in heart failure with preserved ejection fraction: a meta-analysis of randomized controlled trials. Heart Fail Rev. 2019; 24(4):535-547, doi: 10.1007/s10741-019-09774-5.

9. Mediano MFF, Leifer ES, Cooper LS, et al. Influence of baseline physical activity level on exercise training response and clinical outcomes in heart failure: the HF-ACTION trial. JACC Heart Fail. 2018; 6(12): 1011-1019, doi: 10.1016/j.jchf.2018.09.012, indexed in Pubmed: 30497641.
10. Tong TK, McConnell AK, Lin H, et al. "Functional" inspiratory and core muscle training enhances running performance and economy. J Strength Cond Res. 2016;30(10):2942-2951, doi: 10.1519/JSC.0000000000000656, indexed in Pubmed: 25162653.

11. Smart NA, Dieberg G, Giallauria F. Functional electrical stimulation for chronic heart failure: a meta-analysis. Int J Cardiol. 2013; 167(1): 80-86, doi: 10.1016/j.jjcard.2011.12.019, indexed in Pubmed: 22236510.

12. Nakagawa NK, Diz MA, Kawauchi TS, et al. Risk factors for inspiratory muscle weakness in chronic heart failure. Respir Care. 2020; 65(4): 507-516, doi: 10.4187/respcare.06766, indexed in Pubmed: 31822596.

13. Lin SJ, McElfresh J, Hall B, et al. Inspiratory muscle training in patients with heart failure: a systematic review. Cardiopulm Phys Ther J. 2012; 23(3): 29-36, indexed in Pubmed: 22993500.

14. Karavidas A, Driva M, Parissis JT, et al. Functional electrical stimulation of peripheral muscles improves endothelial function and clinical and emotional status in heart failure patients with preserved left ventricular ejection fraction. Am Heart J. 2013; 166(4): 760-767, doi: 10.1016/j. ahj.2013.06.021, indexed in Pubmed: 24093858.

15. DobsákP, Nováková M, Fiser B, et al. Electrical stimulation of skeletal muscles. An alternative to aerobic exercise training in patients with chronic heart failure? Int Heart J. 2006; 47(3): 441-453, doi: 10.1536/ihj.47.441, indexed in Pubmed: 16823250.

16. Quittan $M$, Wiesinger GF, Sturm B, et al. Improvement of thigh muscles by neuromuscular electrical stimulation in patients with refractory heart failure: a single-blind, randomized, controlled trial. Am J Phys Med Rehabil. 2001;80(3):206-214, doi: 10.1097/00002060-200103000-00011, indexed in Pubmed: 11237275.

17. Moher D, Liberati A, Tetzlaff J, et al. PRISMA Group. Preferred reporting items for systematic reviews and meta-analyses: the PRISMA Statement. Open Med. 2009; 3(3): e123-e130, indexed in Pubmed: 21603045.

18. Higgins JPT, Altman DG, Gøtzsche PC, et al.Cochrane Bias Methods Group, Cochrane Statistical Methods Group. The Cochrane Collaboration's tool for assessing risk of bias in randomised trials. BMJ. 2011; 343: d5928, doi: 10.1136/bmj.d5928, indexed in Pubmed: 22008217.

19. Edelmann $F$, Gelbrich $G$, Düngen $H D$, et al. Exercise training improves exercise capacity and diastolic function in patients with heart failure with preserved ejection fraction: results of the Ex-DHF (Exercise training in Diastolic Heart Failure) pilot study. J Am Coll Cardiol. 2011; 58(17): 1780-1791, doi: 10.1016/j.jacc.2011.06.054, indexed in Pubmed: 21996391.

20. Nolte K, Herrmann-Lingen C, Wachter R, et al. Effects of exercise training on different quality of life dimensions in heart failure with preserved ejection fraction: the Ex-DHF-P trial. Eur J Prev Cardiol. 2015; 22(5): 582-593, doi: 10.1177/2047487314526071, indexed in Pubmed: 24627449.

21. Kitzman $D$, Brubaker $P$, Morgan $T$, et al. Exercise training in older patients with heart failure and preserved ejection fraction. Circ Heart Fail. 2010; 3(6): 659-667, doi: 10.1161/circheartfailure.110.958785.

22. Smart NA, Haluska $B$, Jeffriess $L$, et al. Exercise training in heart failure with preserved systolic function: a randomized controlled trial of the effects on cardiac function and functional capacity. Congest Heart Fail. 2012; 18(6): 295-301, doi: 10.1111/j.1751-7133.2012.00295.x, indexed in Pubmed: 22536983.

23. Maldonado-Martín S, Brubaker PH, Eggebeen J, et al. Association between 6-minute walk test distance and objective variables of functional capacity after exercise training in elderly heart failure patients with preserved ejection fraction: a randomized exercise trial. Arch Phys Med Rehabil. 2017; 98(3): 600-603, doi: 10.1016/j.apmr.2016.08.481, indexed in Pubmed: 27693420.

24. Fu TC, Yang NI, Wang $\mathrm{CH}$, et al. Aerobic interval training elicits different hemodynamic adaptations between heart failure patients with preserved and reduced ejection fraction. Am J Phys Med Rehabil. 2016; 95(1): 15-27, doi: 10.1097/PHM.0000000000000312, indexed in Pubmed: 26053189.

25. Kitzman DW, Brubaker PH, Herrington DM, et al. Effect of endurance exercise training on endothelial function and arterial stiffness in older patients with heart failure and preserved ejection fraction: a randomized, controlled, single-blind trial. J Am Coll Cardiol. 2013; 62(7):584-592, doi: 10.1016/j.jacc.2013.04.033, indexed in Pubmed: 23665370.

26. Gary RA, Sueta CA, Dougherty $M$, et al. Home-based exercise improves functional performance and quality of life in women with diastolic 
heart failure. Heart Lung. 2004; 33(4): 210-218, doi: 10.1016/j.hrtlng.2004.01.004, indexed in Pubmed: 15252410.

27. Alves AJ, Ribeiro F, Goldhammer E, et al. Exercise training improves diastolic function in heart failure patients. Med Sci Sports Exerc. 2012; 44(5): 776-785, doi: 10.1249/MSS.0b013e31823cd16a, indexed in Pubmed: 22005747.

28. Haykowsky MJ, Brubaker PH, Stewart KP, et al. Effect of endurance training on the determinants of peak exercise oxygen consumption in elderly patients with stable compensated heart failure and preserved ejection fraction. J Am Coll Cardiol. 2012; 60(2): 120-128, doi: 10.1016/j. jacc.2012.02.055, indexed in Pubmed: 22766338.

29. Palau $P$, Domínguez $E$, López $L$, et al. Inspiratory muscle training and functional electrical stimulation for treatment of heart failure with preserved ejection fraction: the TRAINING-HF trial. Rev Esp Cardiol (Engl Ed). 2019; 72(4): 288-297, doi: 10.1016/j.rec.2018.01.010, indexed in Pubmed: 29551699.

30. Palau $P$, Domínguez $E$, López $L$, et al. Effects of inspiratory muscle training in patients with heart failure with preserved ejection fraction. Eur J Prev Cardiol. 2014; 21(12): 1465-1473, doi: 10.1177/2047487313498832, indexed in Pubmed: 23864363.

31. Gomes-Neto M, Durães AR, Conceição LS, et al. Effect of aerobic exercise on peak oxygen consumption, VE/VCO slope, and health-related quality of life in patients with heart failure with preserved left ventricular ejection fraction: a systematic review and meta-analysis. Curr Atheroscler Rep. 2019; 21(11):45, doi: 10.1007/s11883-019-0806-6, indexed in Pubmed:31707525.

32. Leggio M, Fusco A, Loreti $C$, et al. Effects of exercise training in heart failure with preserved ejection fraction: an updated systematic literature review. Heart Fail Rev. 2020; 25(5): 703-711, doi: 10.1007/s10741-019-09841-x, indexed in Pubmed: 31399956.

33. Rundqvist $L$, Engvall J, Faresjö $M$, et al. Left ventricular diastolic function is enhanced after peak exercise in endurance-trained adolescents as well as in their non-trained controls. Clin Physiol Funct Imaging. 2018 [Epub ahead of print], doi: 10.1111/cpf.12534, indexed in Pubmed: 29947056.

34. Borlaug BA. The pathophysiology of heart failure with preserved ejection fraction. Nat Rev Cardiol. 2014; 11(9): 507-515, doi: 10.1038/nrcardio.2014.83, indexed in Pubmed: 24958077.
35. Nagueh SF, Appleton CP, Gillebert TC, et al. Recommendations for the evaluation of left ventricular diastolic function by echocardiography. J Am Soc Echocardiogr. 2009; 22(2): 107-133, doi: 10.1016/j.echo.2008.11.023, indexed in Pubmed: 19187853.

36. Shuai $X X$, Chen $Y Y, L u Y X$, et al. Diagnosis of heart failure with preserved ejection fraction: which parameters and diagnostic strategies are more valuable? Eur J Heart Fail. 2011; 13(7): 737-745, doi: 10.1093/eurjhf/hfr053, indexed in Pubmed: 21602549.

37. Mitter SS, Shah SJ, Thomas JD. A test in context: E/A and E/e' to assess diastolic dysfunction and LV filling pressure. J Am Coll Cardiol. 2017; 69(11): 1451-1464, doi: 10.1016/j.jacc.2016.12.037, indexed in Pubmed: 28302294.

38. Silbiger JJ. Pathophysiology and echocardiographic diagnosis of left ventricular diastolic dysfunction. J Am Soc Echocardiogr. 2019; 32(2):216-232. e2, doi: 10.1016/j.echo.2018.11.011, indexed in Pubmed: 30717860.

39. Fujimoto N, Prasad A, Hastings JL, et al. Cardiovascular effects of 1 year of progressive endurance exercise training in patients with heart failure with preserved ejection fraction. Am Heart J. 2012; 164(6): 869-877, doi: 10.1016/j.ahj.2012.06.028, indexed in Pubmed: 23194487.

40. Borlaug BA, Olson TP, Lam CSP, et al. Global cardiovascular reserve dysfunction in heart failure with preserved ejection fraction. J Am Coll Cardiol. 2010; 56(11): 845-854, doi: 10.1016/j.jacc.2010.03.077, indexed in Pubmed: 20813282.

41. Hellsten $Y$, Nyberg M. Cardiovascular adaptations to exercise training. Compr Physiol. 2015; 6(1): 1-32, doi: 10.1002/cphy.c140080, indexed in Pubmed: 26756625.

42. Neufer PD. The bioenergetics of exercise. Cold Spring Harb Perspect Med. 2018; 8(5): a029678, doi: 10.1101/cshperspect.a029678, indexed in Pubmed: 28490536.

43. Hughes DC, Ellefsen S, Baar K. Adaptations to endurance and strength training. Cold Spring Harb Perspect Med. 2018; 8(6): a029769, doi: 10.1101/cshperspect.a029769, indexed in Pubmed: 28490537.

44. Illi SK, Held U, Frankl, et al. Effect of respiratory muscle training on exercise performance in healthy individuals: a systematic review and meta-analysis. Sports Med. 2012; 42(8): 707-724, doi: 10.1007/BF03262290, indexed in Pubmed: 22765281. 